\title{
Depression from the perspective of modern and Persian medicine
}

\author{
Majid Anushiravani ${ }^{1}$, Ali Akhondpour Manteghi ${ }^{2}$, Ali Taghipur ${ }^{3}$, Mahdi Eslami ${ }^{4}$
}

${ }^{1}$ MD. PhD in Persian Medicine, Assistant Professor, Department of Persian Medicine, School of Persian and Complementary Medicine, Mashhad University of Medical Sciences, Mashhad, Iran

${ }^{2}$ Associate Professor of Psychiatry, Psychiatry and Behavioral Sciences Research Center, Mashhad University of Medical Sciences, Mashhad, Iran

${ }^{3}$ MD. PhD in Epidemiology, Associate Professor, Department of Epidemiology \& Biostatistics, School of Health, Social Determinants of Health Research Center, Cancer Research Center, Mashhad University of Medical Sciences, Mashhad, Iran

${ }^{4}$ MD. PhD Candidate in Persian Medicine, Department of Persian Medicine, School of Persian and Complementary Medicine, Mashhad University of Medical Sciences, Mashhad, Iran

\section{Type of article: Original}

\begin{abstract}
Background: Depression is one of the five most-common diseases globally, and is expected to be the second leading cause of disability by 2020 and its economic and social burden is a major problem worldwide

Objective: The aim of this research was to elucidate the causes and symptoms of depression according to Persian Medicine (PM) and classic medicine.

Methods: In this study, works that were investigated were the Canon of Medicine, Al-Hawi Fi Teb, Kamel AlSina'a, Zakhireh Kharazmshahi and Exir A'azam. Classic medicine sources investigated were Kaplan and Sadock's Synopsis of Psychiatry and related articles in the Web of Science, Medline, Scopus, SID and Magiran. Research was done from January through April 2017, using keywords.

Results: To explain the biologic causes, various factors including humoral dystemperaments, cold and hot dystemperaments of chief organs especially heart and brain and some qualitative and quantitative changes in medical spirit should be considered. According to manuscripts some mental-emotional events in life can cause these changes. They independently may cause depression. Semiologically some symptoms and signs happen consequently to in the above-mentioned causes which are in common with depression signs and symptoms including grief, crying, low libido, weight loss, appetite and sleep disorders, exhaustion, slow cognitive processing, indecisiveness and willingness to die.

Conclusion: The major biological causes of depression is a group of dystemperamental syndromes (hot, cold, dry, and wet) on different bodily structures (humors, organs, and spirits). So preventive and therapeutic strategies for depression couldn't be the same for all patients and the treatment should be designed according to the exact diagnosis.
\end{abstract}

Keywords: Major Depressive Disorder, Temperament, Persian Medicine

\section{Introduction}

Mood disorders are the most common psychiatric disorders. Mood is defined as a pervasive and sustained emotion or feeling tone that influences a person's behavior and his/her perception of the world. Depression is classified as a mood disorder. Depressed patients, experience loss of energy and interest, guilt, difficult concentration, loss of appetite and thoughts of death or suicide. Other signs and symptoms include changes in activity level, cognitive abilities, speech and biological functions (such as sleep pattern and sexual activity). These changes almost always impair the interpersonal, social and occupational functioning of the patient (1). Depression is one of the five most-

\section{Corresponding author:}

Dr. Mahdi Eslami, Department of Persian Medicine, School of Persian and Complementary Medicine, Mashhad University of Medical Sciences. Azadi Square, Mashhad-Iran.

Tel: +98.9153201459, Fax: +98.5138829279, E-mail: esl_mah@yahoo.com

Received: May 10, 2017, Accepted: July 10, 2017, Published: February 2018

iThenticate screening: July 04, 2017, English editing: September 12, 2017, Quality control: October 12, 2017

This article has been reviewed / commented by three experts

(C) 2018 The Authors. This is an open access article under the terms of the Creative Commons Attribution-NonCommercialNoDerivs License, which permits use and distribution in any medium, provided the original work is properly cited, the use is non-commercial and no modifications or adaptations are made. 
common disorders globally and is expected to be the second leading cause of disability by 2020 (2). It has a high personal, economic and social cost $(3,4)$. Recent surveys have shown that major depressive disorder has the highest lifetime prevalence (almost 17\%) of any psychiatric disorder. The annual incidence rate of major depressive disorder is $1.59 \%$ ( $1.89 \%$ for men and $1.1 \%$ women) (5). The World Health Organization states that 350 million people suffer from the disease worldwide according to estimates; the current prevalence reported $2 \%$ in China, $2.9 \%$ in Japan, $6.6 \%$ in the United States of America and $8.3 \%$ in Ukraine (6). The results of a systematic review of 44 articles found that the current prevalence of major depressive disorder in Iran is $4.1 \%$ (3.1\% to $5.1 \%)$ and the risk in women is 1.95 -fold greater (7). The explanatory model of modern medicine for depression has led to some pharmaceutical interventions which their efficacy and safety hasn't been yet so sufficient. It appears that other schools of medicine might provide wider perspectives to explain the biologic causes of depression. One of the most ancient holistic medical schools is PM which characterized by expanded manuscripts by sage philosopher/physicians like Avicenna since more than 1000 years before. This old medical school considers the whole mental/emotional and physical aspects of human being in order to maintain health. Depression is dominantly and mistakenly has been attributed to a melancholic dystemperament; or considered to be equivalent to melancholia by uneducated traditional practitioners; which led to neglect the wide and various probable causes. The objective of this study was to elucidate depression in PM considering the conformity assessment with classic medicine, including the following items: The etiology, the semiology, and therapeutics of depression

\section{Material and Methods}

\subsection{Identifying relevant publications}

In this study Persian medical manuscripts were explored using the qualitative research approach. our chosen resources were Canon of Medicine, al-hawi fi teb, kamel al-sina'a, tafrih al-qoloob, zakhireh kharazmshahi, sharh alasbab, Tibb e Akbari, mofarrih al-qoloob, darooha-yi qalbi, makhzan al-adviah and exir azam. Classic medicine sources were Kaplan and Sadock's Synopsis of Psychiatry 11th ed. 2015 and related articles in the Web of Science, Medline, Scopus, Scientific Information Database (SID) and Magiran databases.

\subsection{Search strategy}

This research was carried out from January to April 2017. In PM sources we searched the Persian/Arabic words and terms such as Andooh (sadness), Ghamm (sadness), Hamm (worry), Farah (happiness), Soo-e- mizaj-e-Rooh (spirit dystemperament), Malikhoolia (Melancholia), etc. in classic sources the words and phrases including Mood and Affective disorder, Major Depressive Disorder, Depression, Unipolar Depression, Depressive Syndromes, Endogenous Depressions, Neurotic Depressions, and Melancholia were searched irrespective of the time of publication.

\section{Results}

\subsection{Etiology}

Based on the manuscripts of PM we found that depression can be caused by different factors as follows:

1) Humoral dystemperament, including melancholic, phlegmatic and choleric dystemperament $(8,9)$

2) Dysfunction of chief organs (heart, brain, and liver) caused mostly by cold dystemperament

3) Active intra-organic axes or collaborative influences between the brain, heart, gut, liver and uterus

4) Alterations in the amount and/or the essence of vital and mental medical spirits. Necessary to remind that according to the PM, medical spirit is a very subtle bodily structure resembled to vapor that emanates from the left ventricle of the heart.

Factors involved in the etiology of depression in classic medicine include biological factors such as biogenic amines, second messengers, intracellular cascades, alteration of hormonal regulation, alteration of sleep neurophysiology, immunological disturbances, genetic factors, psychosocial factors such as life events and environmental stress, personality factors, psychodynamic factors, cognitive distortion and learned helplessness (3). Table 1 and 2 show the causes of depression in PM and causes of depression in classic medicine

\subsection{Semiology}

According to the above-mentioned causes of depression, the following patterns may be seen: The manuscripts indicate that dystemperaments of phlegm, black bile and yellow bile could cause depression. Melancholic dystemperament is characterized by weight loss, insomnia, thick and high-viscous blood, worry, obsession, and nightmares. Symptoms of phlegmatic dystemperament are decreased body temperature, slow pulse, low pulse rate, malaise, lethargy, slow-wittedness, and dreams of water, sea, snow, rain or cold. (10-12). Melancholia can also be considered in elucidating of depression in which an individual's thoughts and ideas do not align with typical functioning. The cause of melancholia according to ancient sages is an increase in black bile in the body or brain. The symptoms of black bile accumulation in the brain is a tendency to obsessive thoughts, despondency, despair, 
isolation and death, fixation of the eyes on the ground or an object, thin face, sunken eyes, and insomnia. (13, 14). Explanations of depression also included heart and brain dystemperament. Cold dystemperament of the heart causes weakness in the body and movements, exhaustion, fatigue, loss of strength, a pale and pasty complexion, cold extremities, yellowness of skin, fear, polysomnia and excessive compassion. Brain cold dystemperament impairs brain function, causes slow-wittedness, lack of imagination, a tendency to fear and boredom which are similar to the symptoms of depression $(15,16)$. Symptoms and signs found in cold dystemperament of vital and mental spirits that may correspond with depression can be classified into two groups: physical and mental/emotional. Physical symptoms include weakness, laxity, cold skin, slow pulse, low pulse and breathing rate, coldness of pulse touch point, pasty appearance, hypersomnia, decreased libido and poor appetite. Mental/emotional symptoms can be listed as confusion, hesitancy, memory loss, fearfulness, touchiness, crying, indifference, and decreased enthusiasm for the future (17). The American Psychiatric Association considers a diagnosis of depression when five (or more) of the following symptoms are present during the same two-week period that represent a change from previous functioning: depressed mood most of the day and on most days; markedly diminished interest or pleasure in all or almost all activities; significant weight loss or gain when not dieting; decrease or increase in appetite; insomnia or hypersomnia; psychomotor agitation or retardation; fatigue or loss of energy; feeling of worthlessness or excessive or inappropriate guilt; diminished ability to concentrate; indecisiveness; recurrent thoughts of death, recurrent suicidal ideation without a specific plan, or a suicide attempt or a specific plan for committing suicide (3).

\subsection{Therapeutics}

Four approaches used in PM to cure diseases including life style reform, nutritional adjustments, using medication, and manual procedures. The purpose of the life style reform is to improve the situation of the six essential affairs to promote the health of an individual. These six affairs indicate the effects of air, food and drink, sleep and wakefulness, detoxification, exercise and rest, and emotional events on health and sickness. A treatment protocol may include all of the therapeutic approaches depending on the causality of sickness, in accordance to the treatment rules. For humeral causes, treatment involves balancing the quantity and quality of humors using diet therapy and natural medications. In temperamental dysfunction of organs, the dystemperament will be treated by diet and medications also. And in general, revitalizing herbs like saffron are used in causes related to vital spirit (10). Classic medical therapies for the biological and psychological aspects of this condition are as follows: pharmacotherapy (norepinephrine, serotonin, norepinephrine and serotonin reuptake inhibitors, pre- and post-synaptic active agents, dopamine reuptake inhibitor, mixed action agents), vagal nerve stimulation, sleep deprivation, phototherapy and psychosocial therapy (cognitive, interpersonal, behavior, psychoanalytically-oriented and family therapy) (3).

Table 1. Causes of depression in PM

\begin{tabular}{|l|l|l|l|}
\hline Humoral dystemperament & Dysfunction of main organs & Active intra-organic axes & Alterations in vital/mental spirits \\
\hline Melancholic & Heart & Heart-Brain axis & Quantitative changes \\
\hline Phlegmatic & Brain & Gut-Brain axis & Qualitative changes \\
\hline Choleric & Liver & Heart-Brain-liver axis & \\
\hline & & Uterus-Heart axis & \\
\hline
\end{tabular}

Table 2. Causes of depression in classic medicine

\begin{tabular}{|rl|}
\hline Biological factors \\
\hline 1. & Biogenic amines (norepinephrine, serotonin, dopamine, other neurotransmitter disturbances) \\
\hline 2. & Second messengers and intracellular cascades \\
\hline 3. & Alterations of hormonal regulation (thyroid axis activity, growth hormone, prolactin) \\
\hline 5. & Alterations of sleep neurophysiology \\
\hline
\end{tabular}

\section{Discussion}

\subsection{Etiology}

Our exploration of PM manuscripts showed that the causes of depression do not fall only into the domain of brain. The heart, with its wide functional scope, has a significant role in the etiology of this condition. PM believes that the origin and formation of the medical spirit is in the heart (18-20), followed by the brain and liver and a specific temperament creates in spirit according to the locus $(17,21)$. As a supportive evidence we can point to the role of oxytocin in emotional status which is secreted by the heart and its effects have been taken into account in the treatment of mental illnesses, including depression $(22,23)$ congregations of oxytocin have also been observed in extra-brain cells, in some other organs of the body (24). Evidence and recent studies indicates that the definition of depression has changed and it is considered a systemic disorder. A meta-analysis by Sara Fernández et al. has elucidated what happens to the organism in an individual suffering from depression. Researchers have concluded 
that depression is more than a mental disorder. It causes important alterations in oxidative stress and should be considered a systemic disorder because it affects the whole organism (25).

\subsection{Semiology}

The results of a comparative study of symptoms of depression in classic medicine and PM are summarized in Table 3. The table indicates that the locus of depression according to PM is the heart, brain, vital spirit or other where. Depressive symptoms based on DSM5 show that some symptoms are fully matched and some partially.

Table 3. Partial correspondence of depression symptoms based on DSM5 and PM

\begin{tabular}{|c|c|c|c|c|c|}
\hline No & $\begin{array}{l}\text { Symptoms of depression } \\
\text { based on DSM5 }\end{array}$ & $\begin{array}{l}\text { Symptoms of heart } \\
\text { cold } \\
\text { dystemperament }\end{array}$ & $\begin{array}{l}\text { Symptoms of } \\
\text { brain cold } \\
\text { dystemperament }\end{array}$ & $\begin{array}{l}\text { Symptoms of vital spirit } \\
\text { cold dystemperament }\end{array}$ & $\begin{array}{l}\text { Symptoms of } \\
\text { melancholia }\end{array}$ \\
\hline 1 & Depressed mood & Sorrow & & Touchiness, crying & Crying, sorrow \\
\hline 2 & $\begin{array}{l}\text { Markedly diminished } \\
\text { interest or pleasure }\end{array}$ & & & Low desire and wish & \\
\hline \multirow[t]{4}{*}{3} & $\begin{array}{l}\text { Significant weight loss } \\
\text { when not dieting }\end{array}$ & & & & Slimness \\
\hline & Weight gain & & & & \\
\hline & Decrease in appetite & & & Anorexia & \\
\hline & Increase in appetite & & & & \\
\hline \multirow[t]{2}{*}{4} & Insomnia & & Lack of sleep & & Lack of sleep \\
\hline & hypersomnia & Hypersomnia & Hypersomnia & Hypersomnia & \\
\hline \multirow[t]{2}{*}{5} & Psychomotor agitation & & & Perplexity and uncertainty & \\
\hline & Psychomotor retardation & & & & \\
\hline 6 & Fatigue or loss of energy & $\begin{array}{l}\text { Weakness and } \\
\text { laxity }\end{array}$ & $\begin{array}{l}\text { Weakness and } \\
\text { laxity }\end{array}$ & Weakness and laxity & $\begin{array}{l}\text { Weakness and } \\
\text { laxity }\end{array}$ \\
\hline 7 & $\begin{array}{l}\text { Feelings of worthlessness } \\
\text { or excessive or } \\
\text { inappropriate guilt }\end{array}$ & & & & \\
\hline \multirow[t]{2}{*}{8} & $\begin{array}{l}\text { Diminished ability to } \\
\text { think or concentrate }\end{array}$ & unpleasant thoughts & $\begin{array}{l}\text { Impairment of } \\
\text { brain functions }\end{array}$ & Slow-wittedness & \\
\hline & Indecisiveness & & & Doubt & \\
\hline 9 & $\begin{array}{l}\text { Recurrent thoughts of } \\
\text { death, recurrent suicidal } \\
\text { ideation }\end{array}$ & & & & Willing to die \\
\hline
\end{tabular}

\subsection{Therapeutics}

Biological causes of depression have various types and subtypes, and any therapeutic intervention should be based on the exact understanding of causes. Etiologic-based treatment is also necessary in classic medicine, but according to PM the etiologic patterns differ in their semiologic appearances. That is why depressed patients show contrary signs and symptoms such as hypersomni vs. insomnia, increase in appetite vs. loss of appetite, psychomotor agitation vs. psychomotor retardation. Depression treatment should vary to meet the conflicting symptom to be effective and avoid serious side effects. It could be considered as one of the difference between modern medicine and PM in their therapeutic approach to depressive disorders.

\section{Conclusions}

Based on the PM literature depression should be considered as a spectrum of some multi-dimensional disorders that stem from different roots causes. Misleadingly, depression has been supposed as a disease of melancholic dystemperament, but in fact it should be attributed to various biological causes. In this context, various biological causes might be involved in the pathogenesis of depression that can be generally presented as various disorders of different corporeal types of the body (humors, organs, and spirits) including dystemperamental syndromes. So it is possible to identify various types of depression based on PM literature, and design holistic medical plans including life style reforms, diet modification, and natural therapies for any etiologic type and semiologic pattern of depression.

\section{Acknowledgments:}

This research was a part of Ph.D. thesis by Dr. Mahdi Eslami, submitted to the School of Persian and Complementary Medicine for fulfillment of the degree of Ph.D. in Persian Medicine, and supported by Mashhad University of Medical Sciences. 


\section{Conflict of Interest:}

There is no conflict of interest to be declared.

\section{Authors' contributions:}

All authors contributed to this project and article equally. All authors read and approved the final manuscript.

\section{References:}

1) Sadock B, Sadock V. Kaplan \& Sadock's Synopsis of Psychiatry [f. Rezaee, trans]. 1. 11th ed. New York: Lippincott Williams \& Wilkins 2015.

2) Rezaee M, Hedayati A, Naghizadeh M, Farjam M, Sabet H, Paknahad M. Correlation between Happiness and Depression According to Beck Depression and Oxford Happiness Inventory among University Students. Galen Medical Journal. 2016; 5(2): 75-81.

3) Dwyer AV, Whitten DL, Hawrelak JA. Herbal medicines, other than St. John's Wort, in the treatment of depression: a systematic review. Altern Med Rev. 2011; 16(1): 40-9. PMID: 21438645.

4) Maughan B, Collishaw S, Stringaris A. Depression in Childhood and Adolescence. J Can Acad Child Adolesc Psychiatry. 2013; 22(1): 35-40. PMID: 23390431, PMCID: PMC3565713.

5) Sadock B, Sadock V. Kaplan \& Sadock's Synopsis of Psychiatry. 1. 11th ed. New York: Lippincott Williams \& Wilkins; 2015.

6) WHO. Depression. 2012. Available from: http://www.who.int/mediacentre/factsheets/fs369/en/.

7) Sadeghirad B, Haghdoost AA, Amin-Esmaeili M, Ananloo ES, Ghaeli P, Rahimi-Movaghar A, et al Epidemiology of major depressive disorder in iran: a systematic review and meta-analysis. Int J Prev Med. 2010; 1(2): 81-91. PMID: 21566767, PMCID: PMC3075476.

8) Nazari M, Taghizadeh A, Orafaei H, Rakhshandeh H, Bazzaz MM, Shokri J, et al. Nausea and vomiting in Iranian Traditional Medicine based on Avicenna's viewpoint. Electron physician. 2015; 7(2): 1047-53. doi: 10.14661/2015.1047-1053. PMID: 26120413, PMCID: PMC4477764.

9) Miraj S, Alesaeidi S, Kiani S. A systematic review of the relationship between dystemprament (sue Mizaj) and treatments and management of diseases (Ilaj and Eslah-e-Mizaj). Electron physician. 2016; 8(12): 3378-84. doi: 10.19082/3378. PMID: 28163851, PMCID: PMC5279969.

10) Nafisi A. Nafisi Dictionary. Tehran: Khayyam; 1963.

11) Dehkhoda A. Dehkhoda Dictionary. Tehran: University of Tehran Press; 1998.

12) Jorjani I. Al-Aghraz Al-Tebbieh val Mabahess Al-Alaiia. Tehran: Iranian Culture Foundation; 1966.

13) Jorjani I. Zakhireh Kharazmshahi. Qom: Ehya'e tibbe tabiee; 2012.

14) Rhazes. Al-Hawi fi Teb. karaj: Al-Hawi Pharmacy Institute; 2001.

15) Arzani M. Mufarrih al-qulub. Tehran: Alma'ee; 2012.

16) A'azam khan Chashti M. Exeer A'azam. Tehran: Iran University of Medical Sciences; 2008.

17) Anushiravani M. Contemplation and explanation of Vital and Mental Spirits in the literature of Traditional Persian Medicine, And a clinical study on it. Tehran: Tehran University of Medical Sciences; 2013.

18) Parviz M, Anushiravani M, Keshavarz M. The Relationship between Vital Spirit and Fevers in the "Canon of Medicine": A Probable Solution for the Controversy over Stress-Induced Hyperthermia. Iran J Public Health. 2013; 42(9): 1073-4. PMID: 26060671, PMCID: PMC4453889.

19) Avicenna. Canon of Medicine. Kashan: Morsal; 2008.

20) Musavi M. Daruha-yi Qalbi. Tehran: Tehran University; 2004.

21) Ahwazi A. kamel al-Sina'a. Qom: Jalal al-Din; 2009.

22) Kain D. Could "Love Hormone" Help Treat Depression? Center USDN. 2012. Available from: http://ucsdnews.ucsd.edu/pressrelease/could_love_hormone_help_treat_depression/.

23) Ji H, Su W, Zhou R, Feng J, Lin Y, Zhang Y, et al. Intranasal oxytocin administration improves depressionlike behaviors in adult rats that experienced neonatal maternal deprivation. Behav Pharmacol. 2016; 27(8): 689-96. doi: 10.1097/FBP.0000000000000248. PMID: 27644094.

24) Scantamburlo G, Hansenne M, Geenen V, Legros JJ, Ansseau M. Additional intranasal oxytocin to escitalopram improves depressive symptoms in resistant depression: an open trial. Eur Psychiatry. 2015; 30(1): 65-8. doi: 10.1016/j.eurpsy.2014.08.007. PMID: 25282363.

25) Depression is not just a mental illness, it affects the whole organism 2016. Available from: http://neurosciencenews.com/depression-systemic-illness-3775/. 